\title{
SYNGE-BEIL AND RIEMANN-JACOBI JET STRUCTURES WITH APPLICATIONS TO PHYSICS
}

\author{
VLADIMIR BALAN
}

Received 6 November 2002

\begin{abstract}
In the framework of geometrized first-order jet approach, we study the SyngeBeil generalized Lagrange jet structure, derive the canonic nonlinear and Cartan connections, and infer the Einstein-Maxwell equations with sources; the classical ansatz is emphasized as a particular case. The Lorentz-type equations are described and the attached Riemann-Jacobi structures for two certain uniparametric cases are presented.
\end{abstract}

2000 Mathematics Subject Classification: 58A20, 58A30, 53B15, 53B50, 53C80.

1. Preliminaries. Let $\left(T, h_{\alpha \beta}\right)$ and $\left(M, \gamma_{i j}\right)$ be two $\mathscr{C}^{\infty}$ pseudo-Riemannian manifolds of dimensions $m$ and $n$, respectively. We denote by $\zeta=\left(E=j^{1}(T\right.$, $M), \pi, T \times M)$ the first-order jet bundle of mappings $\varphi: T \rightarrow M$, with the local coordinates

$$
\left(t^{\alpha}, x^{i}, y^{A}\right)_{(\alpha, i, A) \in I_{*}} \equiv\left(y^{\mu}\right)_{\mu \in I} \text {. }
$$

Throughout the paper, we consider the sets

$$
\begin{gathered}
I=I_{h} \cup I_{v}, \quad I_{h}=I_{h_{1}} \cup I_{h_{2}}, \quad I_{h_{1}}=\overline{1, m}, \quad I_{h_{2}}=\overline{m+1, m+n}, \\
I_{v}=\overline{m+n+1, N, \quad I_{*}=I_{h_{1}} \times I_{h_{2}} \times I_{v}, \quad N=m+n+m n ;}
\end{gathered}
$$

the indices will implicitly take the values

$$
\alpha, \beta, \ldots \in I_{h_{1}}, \quad i, j, \ldots \in I_{h_{2}}, \quad A, B, \ldots \in I_{v}, \quad \lambda, \mu, \ldots \in I .
$$

For $A=m+n+n(i-m-1)+\alpha$, we will denote $A \equiv\left(\begin{array}{l}i \\ \alpha\end{array}\right)$ and $y^{A} \equiv x^{\left(\begin{array}{l}i \\ \alpha\end{array}\right)}=$ $\partial x^{i} / \partial t^{\alpha}$.

We endow $E$ with the sub-Riemannian Synge-Beil metric (see [9])

$$
\tilde{g}_{A B} \equiv \tilde{g}_{\left(\begin{array}{c}
i \\
\alpha
\end{array}\right)\left(\begin{array}{c}
j \\
\beta
\end{array}\right)}=h^{\alpha \beta}(t) g_{i j}(t, x, y),
$$

where

$$
g_{i j}(t, x, y)=\gamma_{i j}(x)+\varepsilon U_{i}(t, x, y) U_{j}(t, x, y), \quad \forall i, j \in I_{h_{2}}, \varepsilon \in\{ \pm 1\},
$$


and $U_{i}(t, x, y)$ is a distinguished 1-form on $E$ (see [1]). We call $(E, \tilde{g})$ the SyngeBeil (SB) jet model. The inverse of $g_{i j}$ is $g^{i j}=\gamma^{i j}-\varepsilon \Theta U^{i} U^{j}$, where $U^{i}=\gamma^{i j} U_{j}$, $\Theta=\left(1+U_{*}\right)^{-1}$, and the star index denotes transvection with $U^{i}$.

We remark the important particular Synge-Beil uniparametric (SBU) autonomous normalized case, where $m=1, s=t^{1}=t$, and $h_{11}=1$, for which we can use the Finsler-Lagrange tangent space notations from [5]. Shifting the indices left by one unit (hence, $I_{h_{2}}=\overline{1, n}, I_{v}=\overline{n+1,2 n}$ ), we have $y^{A} \equiv y^{\left(\begin{array}{l}i \\ 1\end{array}\right) \stackrel{\text { not }}{=}} y^{i}$. In this case, considering

$$
U_{i}=\left[k\left(1-n^{-2}(x, y)\right)\right]^{1 / 2} y_{i}, \quad k>0,
$$

we encounter three important extensively studied cases.

(I) The Synge classical framework (see [10]), obtained for $\varepsilon=1$ and $k=1$, where $y_{i}=\gamma_{i j} y^{j}, n(x, y)$ is the refraction index of relativistic optics (see $[7,9])$, and the direction $y=X(x)$ is provided by a vector field $X \in \mathscr{L}(M)$.

(II) If the potentials $U_{i}$ in (1.5) are 0-homogeneous relative to $y$, in the limit case $n \rightarrow \infty$ with $\varepsilon=1, k \in \mathscr{F}(M)$, we have

$$
g_{i j}(x, y)=\gamma_{i j}(x)+k \cdot U_{i}(x, y) U_{j}(x, y)
$$

and we may consider the Finsler fundamental function $F=\sqrt{L}$, where

$$
L=g_{i j}(x, y) y^{i} y^{j}
$$

This is the relativistic Beil-type metric (see [3, 4]) with the two intensively studied subcases

$$
U_{i} \in\left\{\left(\gamma_{j k} v^{j} v^{k}\right)^{-1 / 2} v_{i},\left(s_{j}(x) v^{j}\right)^{-1} v_{i}\right\}
$$

where $s \in \mathscr{L}^{*}(M)$, and $v \in X^{*}(T M)$ is 0-homogeneous in $y$.

(III) The generalized Lagrange model of relativistic optics studied by Miron and Kawaguchi (see [6, 7]) is obtained as limit case $n \rightarrow \infty$ with $\varepsilon=1, k=1 / c^{2}$ ( $c=$ speed of light), where the metric is

$$
g_{i j}(x, y)=\gamma_{i j}(x)+c^{-2} \cdot y_{i} y_{j}, \quad \forall i, j \in I_{h_{2}} .
$$

Considering the general SB-jet case (1.5), we can fix a priori on $E$ a nonlinear connection $N=\left\{N_{\mu}^{A}\right\}_{\mu \in I_{h}, A \in I_{v}}$ of coefficients

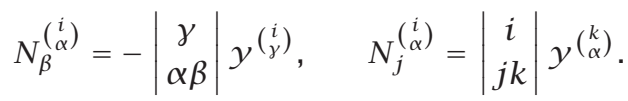


However, an open question (see [9]) addresses the physical significance of choosing an alternative target-nonlinear connection coefficients provided by the spray attached to the Lagrangian

$$
L=\tilde{g}_{A B} y^{A} y^{B},
$$

given by $\tilde{N}_{j}^{\left(\begin{array}{c}i \\ \alpha\end{array}\right)}=N_{j}^{\left(\begin{array}{c}i \\ \alpha\end{array}\right)}+(\varepsilon / 2) g^{i k} \partial_{\alpha}\left(U_{k} U_{j}\right)$.

The fixed nonlinear connection leads to a splitting $T E=H E \oplus V E$, where $V E=\operatorname{Ker} \pi_{*}$, and to the associated local adapted basis of vector fields [1, 9]

$$
\mathscr{B}=\left\{\delta_{\alpha} \equiv \partial_{\alpha}-N_{\alpha}^{A} \delta_{A}, \delta_{i} \equiv \partial_{i}-N_{i}^{A} \delta_{A}, \delta_{A} \equiv \dot{\partial}_{A}=\frac{\partial}{\partial y^{A}}\right\}_{(\alpha, i, A) \in I_{*}} \equiv\left\{\delta_{\mu}\right\}_{\mu \in I}
$$

where $\partial_{\alpha}=\partial / \partial t^{\alpha}, \partial_{i}=\partial / \partial x^{i}$, of dual basis

$$
\begin{aligned}
\mathscr{B}^{*}= & \left\{\delta^{\alpha} \equiv d t^{\alpha}, \delta^{i} \equiv d x^{i},\right. \\
& \left.\delta^{A} \delta y^{A}=d y^{A}+N_{\alpha}^{A} d t^{\alpha}+N_{i}^{A} d x^{i}\right\}_{(\alpha, i, A) \in I_{*}} \equiv\left\{\delta^{\mu}\right\}_{\mu \in I^{*}}
\end{aligned}
$$

For $N$ fixed, a linear connection $\nabla=\left\{L_{\mu \nu}^{\lambda}\right\}_{\lambda, \mu, v \in I}$ in $E$ has the adapted coefficients provided by $\delta^{\lambda}\left(\nabla_{\delta_{\nu}} \delta_{\mu}\right)=L_{\mu \nu}^{\lambda}$ for all $\lambda, \mu, \nu \in I$; these split into $3^{3}=27$ distinct subsets according to the three index subsets $I_{h_{1}}, I_{h_{2}}$, and $I_{v}$.

We endow $E$ with the metric

$$
G=\underbrace{h_{\alpha \beta}(t) d t^{\alpha} \otimes d t^{\beta}}_{h}+\underbrace{g_{i j}(t, x, y) d x^{i} \otimes d x^{j}}_{g}+\underbrace{\tilde{g}_{A B}(t, x, y) \delta y^{A} \otimes \delta y^{B}}_{\tilde{g}}
$$

with $g_{i j}$ given in (1.5). The Cartan linear connection has the four essential sets of coefficients

$$
\begin{aligned}
L_{\beta \gamma}^{\alpha} & =\left|\begin{array}{c}
\alpha \\
\beta \gamma
\end{array}\right|, \quad L_{j k}^{i}=\left|\begin{array}{c}
i \\
j k
\end{array}\right|+\tilde{U}_{j k}^{i}, \\
L_{j \alpha}^{i} & =\frac{\left[\varepsilon\left(U^{i} U_{j}\right)_{; \alpha}-\Theta U^{i} U^{m}\left(U_{m} U_{j}\right)_{; \alpha}\right]}{2}, \\
L_{j A}^{i} & \equiv L_{j\left(\begin{array}{l}
k \\
\alpha
\end{array}\right)}^{i}=\gamma^{i m} U_{\left(\begin{array}{c}
j \\
\alpha
\end{array}\right) k m}-U_{\left(\begin{array}{c}
j \\
\alpha
\end{array}\right) k m} \Theta U^{i} U^{m}
\end{aligned}
$$

with

$$
\begin{aligned}
\tilde{U}_{j k}^{i} & =\gamma^{i m} U_{j k m}-\varepsilon \Theta\left(\gamma_{j k *}-U_{j k *}\right) U^{i}, \\
U_{i j m} & =\frac{\varepsilon\left[\delta_{\{j}\left(U_{m} U_{i\}}\right)-\delta_{m}\left(U_{i} U_{j}\right)\right]}{2}, \\
U_{\left(\begin{array}{c}
i \\
\alpha
\end{array}\right) j m} & =\frac{\varepsilon\left[\delta_{\left(\begin{array}{c}
\{j \\
\alpha
\end{array}\right)}\left(U_{m} U_{i\}}\right)-\delta_{\left(\begin{array}{c}
m \\
\alpha
\end{array}\right)}\left(U_{i} U_{j}\right)\right]}{2}, \\
\gamma_{i j m} & =|i j ; m|=\frac{\left(\partial_{\{j} \gamma_{m i\}}-\partial_{m} \gamma_{i j}\right)}{2},
\end{aligned}
$$


where we denote by $\alpha$ and $k$ the natural covariant derivatives on $\left(T, h_{\alpha \beta}\right)$ and $\left(M, \gamma_{i j}\right)$, respectively, by $\left|\begin{array}{c}\alpha \gamma \\ \beta \gamma\end{array}\right|$ and $|\underset{j k}{i}|$ the Christoffel symbols of the metrics $h$ and $\gamma$, respectively, and $\tau_{[i \cdots j]}=\tau_{i \cdots j}-\tau_{j \cdots i}, \tau_{\{i \cdots j\}}=\tau_{i \cdots j}+\tau_{j \cdots i}$.

The torsion and the curvature of $\nabla$ adapted coefficients are given by

$$
\delta^{\lambda}\left(\mathscr{T}\left(\delta_{\nu}, \delta_{\mu}\right)\right)=T_{\mu \nu}^{\lambda}, \quad \delta^{\lambda}\left(\mathscr{R}\left(\delta_{\nu}, \delta_{\mu}\right) \delta_{\rho}\right)=R_{\rho \mu \nu}^{\lambda}, \quad \forall \lambda, \mu, \nu, \rho \in I .
$$

In the Cartan connection case, the essential associated torsion coefficients are (see [9])

$$
\begin{aligned}
& T_{\alpha j}^{i}=-L_{j \alpha}^{i}, \quad T_{\beta\left(\begin{array}{l}
k \\
\gamma
\end{array}\right)}^{\left(\begin{array}{c}
i \\
\alpha
\end{array}\right)}=-\delta_{\alpha}^{\gamma} L_{j \beta}^{i}, \quad T_{j\left(\begin{array}{l}
k \\
\gamma
\end{array}\right)}^{\left(\begin{array}{c}
i \\
k
\end{array}\right)}=-\delta_{\alpha}^{\gamma} \tilde{U}_{j k}^{i}, \quad T_{j A}^{i}=L_{j A}^{i}, \\
& T_{\left(\begin{array}{l}
j \\
\beta
\end{array}\right)\left(\begin{array}{l}
k \\
\gamma
\end{array}\right)}^{\left(\begin{array}{l}
i \\
\beta
\end{array}\right)}=\delta_{\alpha}^{[\beta} L_{j\left(\begin{array}{c}
k \\
\gamma]
\end{array}\right)}^{i}, \quad T_{\beta \gamma}^{\left(\begin{array}{l}
i \\
\alpha
\end{array}\right)}=-\rho_{\alpha \beta \gamma}^{\delta} y^{\left(\begin{array}{c}
i \\
\delta
\end{array}\right)}, \\
& T_{j k}^{\left(\begin{array}{c}
i \\
\alpha
\end{array}\right)}=\rho_{j k l}^{i} y^{\left(\begin{array}{l}
l \\
\alpha
\end{array}\right),}, \quad T_{\beta j}^{\left(\begin{array}{c}
i \\
\alpha
\end{array}\right)}=0,
\end{aligned}
$$

where $\rho_{\alpha \beta \gamma}^{\delta}$ and $\rho_{j k l}^{i}$ are the curvature components of the metrics $h$ and $\gamma$ respectively. The nonholonomy coefficients $\omega_{\mu \nu}^{\lambda}$ given by $\left[\delta_{\mu}, \delta_{\nu}\right]=\omega_{\mu \nu}^{A} \delta_{A}$, for all $\mu, \nu \in I$, are related to torsion via $T_{\mu \nu}^{\lambda}=L_{[\mu \nu]}^{\lambda}+\omega_{\mu \nu}^{\lambda}$, for all $\lambda, \mu, v \in I$, and the essential curvature $N$-tensor fields (for explicit expressions, see [9]) are

$$
R_{\mu \nu \pi}^{\lambda}=\delta_{[\pi} L_{\mu] v}^{\lambda}+L_{\mu[v}^{\sigma} L_{\sigma \pi]}^{\lambda}+L_{\mu \sigma}^{\lambda} \omega_{v \pi}^{\sigma}
$$

Denoting by $|\alpha| i,, \mid A$, and $\mid \lambda$ the covariant derivations given by $\nabla_{\delta_{\mu}}$, for $\mu \in$ $I_{h_{1}}, I_{h_{2}}, I_{v}$, and $I$, respectively, the Ricci identities for $X \in \mathscr{L}(E)$ and $\theta \in \mathscr{L}^{*}(E)$ are

$$
\begin{gathered}
X_{\mid[\mu \mid v]}^{\lambda}=R_{\sigma \mu \nu}^{\lambda} X^{\sigma}-T_{\mu \nu}^{\sigma} X_{\mid \sigma}^{\lambda}, \\
\theta_{\lambda \mid[\mu \mid v]}=R_{\lambda \mu \nu}^{\sigma} \theta_{\sigma}+T_{\mu \nu}^{\sigma} \theta_{\lambda \mid \sigma}, \quad \forall \lambda, \mu, \nu \in I .
\end{gathered}
$$

The adapted components of the Ricci tensor field are given by $R_{\lambda \mu}=R_{\lambda \mu \nu}^{\nu}$ and the scalar of curvature is $R \equiv G^{\mu v} R_{\lambda \mu v}^{v}=R_{h}+R_{g}+R_{v}$, where

$$
\begin{aligned}
R_{h}=h^{\alpha \beta} \rho_{\alpha \beta \gamma}^{\gamma}, & R_{\mathcal{g}}=\left(\gamma^{i j}-\varepsilon \Theta U^{i} U^{j}\right)\left(\rho_{i j k}^{k}+U_{i j k}^{k}\right), \\
R_{v} & =\tilde{g}^{A B} R_{A B},
\end{aligned}
$$

and $U_{j k l}^{i}=\tilde{U}_{j[k \mid l]}^{i}+\tilde{U}^{m}{ }_{j[k} \tilde{U}_{m l]}^{i}+L_{j\left(\begin{array}{c}m \\ \alpha\end{array}\right)}^{i} \rho_{p k l}^{m} y^{\left(\begin{array}{c}p \\ \alpha\end{array}\right)}$

2. Einstein-Maxwell equations. Denoting by $E_{\mu \nu}=R_{\mu \nu}+(1 / 2) R G_{\mu \nu}$ the Einstein $N$-tensor field, the Einstein equations with sources 


$$
E_{\mu \nu}=\kappa \mathscr{T}_{\mu \nu}, \quad \mu, \nu \in I,
$$

split

$$
\begin{gathered}
R_{\alpha \beta}-\frac{1}{2} R h_{\alpha \beta}=\kappa \mathscr{T}_{\alpha \beta}, \\
R_{i j}-\frac{1}{2} R g_{i j}=\kappa \mathscr{T}_{i j}, \\
R_{A B}-\frac{1}{2} R g_{A B}=\kappa \mathscr{T}_{A B}, \\
0=\mathscr{T}_{\alpha i}, \quad 0=\mathscr{T}_{\alpha A}, \\
R_{i \alpha}=\kappa \mathcal{T}_{i \alpha}, \quad R_{A \alpha}=\kappa \mathcal{T}_{A \alpha}, \\
R_{i A}=\kappa \mathcal{T}_{i A}, \quad R_{A i}=\kappa \mathcal{T}_{A i},
\end{gathered}
$$

where $\mathscr{T}=\mathscr{T}_{\mu \nu} \delta^{\mu} \otimes \delta^{\nu} \in \mathscr{T}_{2}^{0}(E)$ is the energy-momentum tensor field and $\kappa$ is the cosmological constant. They satisfy the conservation laws

$$
E_{v \mid \mu}^{\mu}=\kappa \mathscr{T}_{v \mid \mu}^{\mu}, \quad \forall \mu \in I=I_{h_{1}} \cup I_{h_{2}} \cup I_{v},
$$

where the indices are raised by means of the metric $G$ in (1.15).

We note that for $U_{i} \equiv 0$, the Einstein equations reduce to the classical ones on $(T \times M, h+g)$. Also, if one considers the extended electromagnetic 2-form

$$
F=F_{A \mu} \delta y^{A} \wedge \delta y^{\mu}
$$

then the Lagrangian density $\mathscr{L}=\left(L+F^{\lambda \mu} F_{\lambda \mu}\right) \sqrt{\operatorname{det} h}$ with $L$ given in (1.12) provides by the Hilbert-Palatini variation the Einstein equations of the form (2.1) with the energy-momentum tensor field

$$
T_{\mu \nu}=F_{\mu \rho} F_{\mu}^{\rho}-\frac{1}{4} G_{\mu \nu} F^{\rho \pi} F_{\rho \pi}, \quad \mu, \nu \in I .
$$

In the extended potential Miron-Tatoiu approach [8], the electromagnetic tensor field $F$ has the essential components

$$
\begin{aligned}
& F_{A \beta} \equiv F_{\left(\begin{array}{c}
i \\
\alpha
\end{array}\right) \beta}=\frac{1}{2}\left(h^{\alpha \gamma} g_{i k} y^{\left(\begin{array}{c}
k \\
{[\gamma}
\end{array}\right)}\right)_{\mid \beta]}, \\
& F_{A j} \equiv F_{\left(\begin{array}{c}
i \\
\alpha
\end{array}\right) j}=\frac{1}{2} d_{\left.\left(\begin{array}{c}
i \\
\alpha
\end{array}\right) j\right]}=\left(\gamma_{[i k} \tilde{U}_{m j]}^{k}+\varepsilon U_{[i} \tilde{U}_{m j]}^{k} U_{k}\right) h^{\alpha \beta} y^{\left(\begin{array}{c}
m \\
\beta
\end{array}\right)}, \\
& F_{A B} \equiv F_{\left(\begin{array}{c}
i \\
\alpha
\end{array}\right)\left(\begin{array}{c}
j \\
\beta
\end{array}\right)}=\frac{1}{2} \tilde{g}_{\left(\begin{array}{c}
{[i} \\
\alpha
\end{array}\right) C} y_{\mid\left(\begin{array}{c}
j] \\
\beta
\end{array}\right)}^{C}=\frac{1}{2} U_{\left(\begin{array}{c}
m \\
\beta
\end{array}\right)[j i]} h^{\alpha \gamma} y^{\left(\begin{array}{c}
m \\
\gamma
\end{array}\right),}
\end{aligned}
$$

derived from the deflection tensor fields (detailed in [9])

$$
d_{\mu}^{A}=\delta^{A} \nabla_{\delta_{\mu}} \mathscr{C}, \quad \mu \in I, A \in I_{v},
$$

where $\mathscr{C}=y^{A} \delta_{A}$ is the Liouville field, by lowering the indices and antisymmetrization; where the raising/lowering of the indices are assumed to be 
performed via the metric $G$. The extended electromagnetic tensor field (2.6) satisfies the following theorem.

THEOREM 2.1. The 2-form $F$ is subject to the two sets of the Maxwell extended equations with sources

$$
\begin{aligned}
& \underset{\alpha \beta \gamma}{S} F_{\left(\begin{array}{c}
i \\
\alpha
\end{array}\right) \beta \mid \gamma}=\frac{1}{2} \underset{\alpha \beta \gamma}{S} h^{\alpha \varepsilon} g_{i k} y_{\mid \beta] \mid \gamma}^{\left(\begin{array}{c}
k \\
{[\varepsilon}
\end{array}\right)}, \\
& F_{\left(\begin{array}{c}
i \\
\alpha
\end{array}\right) j \mid \beta}=\frac{1}{2}\left[d_{\left.\left(\begin{array}{c}
{[i} \\
\alpha
\end{array}\right) \beta \mid j\right]}+\left(y_{\left(\begin{array}{c}
m \\
\alpha
\end{array}\right)} L^{m}{ }_{[i \beta}\right)_{\mid j]}-d_{\left(\begin{array}{c}
i \\
\alpha
\end{array}\right) m} L_{j \beta}^{m}\right] \text {, } \\
& F_{\left(\begin{array}{c}
i \\
\alpha
\end{array}\right)\left(\begin{array}{c}
j \\
\beta
\end{array}\right) \mid \gamma}=\frac{1}{2}\left[d_{\left(\begin{array}{c}
{[i} \\
\alpha
\end{array}\right) \gamma \mid\left(\begin{array}{c}
j] \\
\beta
\end{array}\right)}+y_{\left(\begin{array}{c}
m \\
\alpha
\end{array}\right)} \partial_{\left(\begin{array}{c}
{[j} \\
\beta
\end{array}\right)} L_{i] \gamma}^{m}\right. \\
& \left.-\left(d_{\left(\begin{array}{c}
{[i} \\
\alpha
\end{array}\right)\left(\begin{array}{c}
m \\
\varepsilon
\end{array}\right)}+L_{\left[i\left(\begin{array}{c}
m \\
\varepsilon
\end{array}\right)\right.}^{k} y_{\left(\begin{array}{c}
k \\
\alpha
\end{array}\right)}\right) L_{j] \delta}^{m} \delta_{\beta}^{\gamma}\right] \text {, } \\
& \underset{i j k}{S} F_{\left(\begin{array}{c}
i \\
\alpha
\end{array}\right) j \mid k}=-\frac{1}{2} S_{i j k}\left[d_{\left(\begin{array}{c}
i \\
\alpha
\end{array}\right)\left(\begin{array}{c}
m \\
\beta
\end{array}\right)}+L_{i\left(\begin{array}{c}
m \\
\beta
\end{array}\right)}^{p} y_{\left(\begin{array}{c}
p \\
\alpha
\end{array}\right)}\right] \rho_{j k l}^{m} y^{\left(\begin{array}{l}
l \\
\beta
\end{array}\right)}, \\
& S_{i j k}\left[F_{\left(\begin{array}{c}
i \\
\alpha
\end{array}\right) j \mid\left(\begin{array}{c}
k \\
\beta
\end{array}\right)}+F_{\left(\begin{array}{c}
i \\
\alpha
\end{array}\right)\left(\begin{array}{c}
j \\
\beta
\end{array}\right) \mid k}\right]=0, \quad F_{\left(\begin{array}{c}
i \\
\alpha
\end{array}\right)\left(\begin{array}{c}
j \\
\beta
\end{array}\right) \mid\left(\begin{array}{c}
k \\
\gamma
\end{array}\right)} \equiv 0, \\
& \tilde{g}^{B C} F_{B \alpha \mid C}=-4 \pi J_{\alpha}, \quad \tilde{g}^{B C} F_{B i \mid C}=-4 \pi J_{i}, \quad G^{\lambda \mu} F_{A \lambda \mid \mu}=4 \pi J_{A},
\end{aligned}
$$

where we denoted by $J=J_{\mu} \delta^{\mu} \in \mathscr{L}^{*}(E)$ the adapted dual electric current and by $S$ the cyclic summation of the corresponding indices below.

The last Maxwell equations in (2.8) were first derived in [9]. Note that in the SBU case with $U_{i} \equiv 0$, the equations above provide in particular the classical Maxwell equations with sources

$$
S_{i j k} F_{i j ; k}=0, \quad \gamma^{i j} F_{i k ; j}=-4 \pi J_{k} .
$$

3. Extended Lorentz equations. The extended Lorentz equations associated to the SB model are $G_{v \rho}\left(\nabla \mathscr{V}^{\rho} / d s\right)=F_{A v} \mathscr{V}^{A}$ [2]; denoting $F_{A}^{\mu}=G^{\mu v} F_{A v}$, for all $\mu \in I$; they can be rewritten as

$$
\frac{\nabla^{\alpha} \mathscr{V}^{\mu}}{d s}=F_{A}^{\mu_{\mathcal{Q}} A}
$$

where

$$
\begin{gathered}
\mathscr{V}=\mathscr{V}^{\mu} \delta_{\mu}, \\
\left\{\mathscr{V}^{\mu}\right\}_{\mu \in I} \equiv\left(\frac{d t^{\alpha}}{d s}, \frac{d x^{i}}{d s}, \frac{\delta y^{A}}{d s}=\frac{d y^{A}}{d s}+N_{\beta}^{A} \frac{d t^{\beta}}{d s}+N_{j}^{A} \frac{d x^{j}}{d s}\right)_{(\alpha, i, A) \in I_{*}}
\end{gathered}
$$

is the covariant velocity along the trajectory of the moving test-particle

$$
c: J \subset \mathbb{R} \longrightarrow E, \quad c(s)=(t(s), x(s), y(s)), \quad \forall s \in J .
$$

We have denoted $\nabla^{\mathcal{V}^{\mu}} / d s=\delta \mathscr{V}^{\mu} / d s+L_{v \rho}^{\mu} \mathcal{V}^{v} \mathcal{V}^{\rho}$, for all $\mu \in I$, and will further use the dot notation for expressing the $s$-derivation. The explicit Lorentz 
equations were described in [2]. In the SBU case with $U_{i}$ dependent on $x$ only, the electromagnetic tensors (2.6) have the components

$$
F_{A}^{\alpha}=0, \quad F_{A}^{i} \equiv g^{i j} F_{\left(\begin{array}{c}
k \\
1
\end{array}\right) j}=g^{i j} g_{[k l} \tilde{U}_{m j]}^{l} y^{\left(\begin{array}{c}
m \\
1
\end{array}\right)}, \quad F_{A}^{B}=0,
$$

and the Lorentz equations (3.1) reduce to [2]

$$
\begin{aligned}
& \ddot{t}^{\alpha}+\left|\begin{array}{c}
\alpha \\
\beta \gamma
\end{array}\right| \dot{t}^{\beta} \dot{t}^{\gamma}=0, \\
& \ddot{x}^{i}+\left|\begin{array}{c}
i \\
j k
\end{array}\right| \dot{x}^{j} \dot{x}^{k}=g^{i j} g_{[k l} \tilde{U}_{m j]}^{l} y^{\left(\begin{array}{c}
m \\
1
\end{array}\right) \mathscr{V}}\left(\begin{array}{l}
k \\
1
\end{array}\right), \quad \dot{q}^{A}=0,
\end{aligned}
$$

and hence are characterized by constant vertical adapted velocity vector field.

4. Riemann-Jacobi structure and energy-dependent Lagrangians. We further consider in the SBU framework two particular cases.

(I) The Miron-Kawaguchi generalized Lagrange case, for $g$ given by

$$
g_{i j}(x, y)=\gamma_{i j}(x)+k \cdot y_{i} y_{j}
$$

with $k \in \mathscr{F}(M)$, where the Lagrangian (1.12) becomes $\tilde{L}=y_{0}\left(1+k y_{0}\right)$ and the null index denotes transvection with $y$. In this case, the Legendre transformation is given locally by $(x, y) \in T M \rightarrow(x, p) \in T^{*} M, p_{i}=\partial L / \partial y^{i}=$ $2 y_{i}\left(1+2 k y_{0}\right), i=\overline{1, n}$, and is a local diffeomorphism on the set

$$
D=\left\{(x, y) \mid y \neq 0,1+2 k y_{0} \neq 0\right\} \subset T M
$$

The associated Hamiltonian is

$$
H \equiv y^{i} \frac{\partial L}{\partial y^{i}}-L=y_{0}\left(1+3 k y_{0}\right)
$$

and the local Riemann-Jacobi structure $(T U, \hat{g})$ provided by the directional variables $U^{i}=y^{i}$ is defined by the scaled metric

$$
\hat{g}_{i j} \equiv\left(H+\frac{1}{2} U_{*}\right) \delta_{i j}=\frac{3}{2}\left(y_{0}+2 k y_{0}^{2}\right) \delta_{i j},
$$

where $U_{*}=\delta_{i j} U^{i} U^{j}$.

(II) The flat local Lagrange space with potential energy-dependence endowed with Riemann-Jacobi generalized Lagrange metric, with the square-type Lagrangian (see [11])

$$
\hat{L}=\frac{1}{2} \delta^{i j}\left(y_{i}-U_{i}\right)\left(y_{j}-U_{j}\right)=\frac{1}{2} y_{0}+U_{0}+f,
$$

where $f=(1 / 2) U_{*}$ and the indices are raised/lowered by means of the Kronecker flat Euclidean metric. Here, the Hamiltonian is $H=(1 / 2) y_{0}-f$ and 
the Legendre transformation provides momenta as potential shifts of direction $p_{i}=y_{i}-U_{i}$. For obtaining the $h$-paths associated to the Kern nonlinear connection [5, Theorem 7.4.1, page 113], we apply for $g_{i j}=\delta_{i j}, a=c=1 / 2$, and $b=1$ the following lemma.

LEMMA 4.1. Let $\left(M, \gamma_{i j}\right)$ be a (pseudo-)Riemannian space. Then

(a) the spray and the Kern nonlinear connection of the Lagrangian

$$
L^{\prime \prime}=a y_{0}+b U_{0}+c U_{*}, \quad a, b, c \in \mathbb{R}
$$

with $U_{i} \in \mathscr{L}^{*}(M)$, for raising/lowering performed using the metric $\gamma_{i j}$ and $U_{*}=U_{i} U^{i}$, are, respectively, given by

$$
\begin{aligned}
& G^{i}(x, y) \equiv \frac{\gamma^{i j}\left(\dot{\partial}_{j} \partial_{k} L^{\prime \prime} \cdot y^{k}-\partial_{j} L^{\prime \prime}\right)}{4} \\
&=\frac{a}{2} \gamma_{00}^{i}+\frac{1}{4}\left\{b\left[\gamma^{i a}\left(y^{k} U^{j} \partial_{[k} \gamma_{a}\right] j-y_{k} \partial_{a} U^{k}\right)+y^{k} \partial_{k} U^{i}\right] \\
&\left.-c \gamma^{i a}\left(U^{j} U^{k} \partial_{a} \gamma_{j k}+2 U_{j} \partial_{a} U^{j}\right)\right\},
\end{aligned}
$$

where $\gamma_{j k}^{i}$ are the Christoffel symbols of $\gamma_{i j}$ and

$$
N_{j}^{i}(x, y) \equiv \frac{\partial G^{i}}{\partial y^{j}}=a \gamma_{j 0}^{i}+\frac{b}{4}\left[\gamma^{i a}\left(\partial_{[j} \gamma_{a] k} \cdot \gamma_{j k} \cdot \partial_{a} U^{k}\right)+\partial_{j} U^{i}\right]
$$

(b) the Euler-Lagrange equations associated to $L^{\prime \prime}$ are the spray equations

$$
\ddot{x}^{i}+2 G^{i}(x, \dot{x})=0, \quad y^{i}=\dot{x}^{i}, \quad i=\overline{1, n},
$$

and the h-paths are given by

$$
\ddot{x}^{i}+N_{j}^{i}(x, \dot{x}) \dot{x}^{j}=0, \quad y^{i}=\dot{x}^{i}, \quad i=\overline{1, n},
$$

with the spray and nonlinear connection determined above.

Then the Kern spray for $g_{i j}=(1 / 2) \cdot\left(\operatorname{Hess}_{y} L\right)_{i j}=\delta_{i j}$ is

$$
G^{i}=\frac{\delta^{i j}\left(\partial_{[j} U_{k]}^{k}+U_{k} \partial_{j} U^{k}\right)}{4},
$$

and denoting $\Omega_{j k}=\partial_{[j} U_{k]}$, this is rewritten as $G^{i}=\delta^{i j}\left(\Omega_{j 0}+\partial_{j} f\right)$. The associated nonlinear connection is then $N_{j}^{i}=(1 / 4) \Omega_{j}^{i}$, and its autoparallel curves (the $h$-paths) satisfy

$$
\ddot{x}^{i}=\frac{1}{4} \Omega_{j}^{i} \dot{x}^{j}
$$

Then we have the following theorem. 
THEOREM 4.2. The h-paths described by (4.12) are as well:

(a) the extended Lorentz curves (see [2]) particularized to the almost Riemann Lagrange special (ARLS) jet case associated to the flat metric $\delta_{i j}$ and to the potential $U_{i} / 2$;

(b) the solutions of the Lorentz-Udriste force law (see [11]) of the RiemannJacobi-Lagrange structure $\left(M=\mathbb{R}^{n}, \hat{g}_{i j}, 4 N_{j}^{i}\right)$, where $\hat{g}$ is the RiemannJacobi metric

$$
\hat{g}_{i j}=(H+f) \delta_{i j}=\frac{y_{0}}{2} \delta_{i j}
$$

(c) the stationary curves (see [5]) of the reduced Lagrangian $\hat{L}^{\prime}=(1 / 2) y_{0}+$ $U_{0}$.

Proof. For (a) and (b), the Riemann-Jacobi and the flat metrics have null Christoffel symbols; for (c), we apply the lemma for $g_{i j}=\delta_{i j}, a=1 / 2, b=1$, and $c=0$.

ACKNOWLEDGment. The present paper was partially supported by Grant CNCSIS MEN 33784 (182)/23.07.2002.

\section{REFERENCES}

[1] V. Balan, Lagrangian structures in geometrized jet framework, Proc. of the 4th International Conference of the Balkan Society of Geometers, Conference of Geometry and Its Application in Technology, Thessaloniki, June 2002, to appear in BSG Proceedings, Geometry Balkan Press, Bucharest, Romania, pp. 25-30.

[2] V. Balan and K. Trenčevski, Stationary Lorentz curves in first-order jet spaces. Numerical simulation and analytic solutions, Proc. of the International Conference on Differential Geometry: Lagrange and Hamilton Spaces, Iaşi, 2002, to appear.

[3] R. G. Beil, Finsler geometry and a unified field theory, Finsler Geometry (Seattle, Wash, 1995), Contemp. Math., vol. 196, American Mathematical Society, Rhode Island, 1996, pp. 265-272.

[4] _ Notes on a new Finsler metric function, Balkan J. Geom. Appl. 2 (1997), no. 1, 1-6.

[5] R. Miron and M. Anastasiei, Vector Bundles and Lagrange Spaces with Applications to Relativity, Balkan Society of Geometers Monographs and Textbooks, vol. 1, Geometry Balkan Press, Bucharest, 1997.

[6] R. Miron and T. Kawaguchi, Relativistic geometrical optics, Internat. J. Theoret. Phys. 30 (1991), no. 11, 1521-1543.

[7] _ Sur la théorie géométrique de l'optique relativiste [On the geometric theory of relativistic optics], C. R. Acad. Sci. Paris Sér. II Méc. Phys. Chim. Sci. Univers Sci. Terre 312 (1991), no. 6, 593-598 (French).

[8] R. Miron and M. Radivoiovici-Tatoiu, Extended Lagrangian theory of electromagnetism, Rep. Math. Phys. 27 (1989), no. 2, 193-229.

[9] M. Neagu, The generalized metrical multi-time Lagrange space of relativistic geometrical optics, 2000, http://xxx.lanl.gov/abs/math.DG/0011262. 
[10] J. L. Synge, Relativity: The General Theory, Series in Physics, North-Holland Publishing, Amsterdam, 1960.

[11] C. Udrişte, Geometric Dynamics, Mathematics and Its Applications, vol. 513, Kluwer Academic Publishers, Dordrecht, 2000.

Vladimir Balan: Department Mathematics I, Politehnica University of Bucharest, Splaiul Independenței 313, RO-77206 Bucharest, Romania

E-mail address: vba1an@mathem. pub.ro 


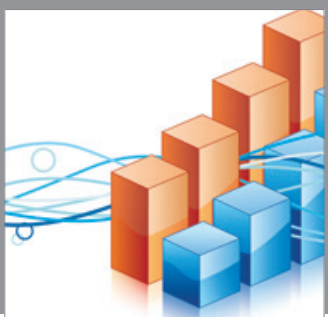

Advances in

Operations Research

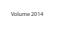

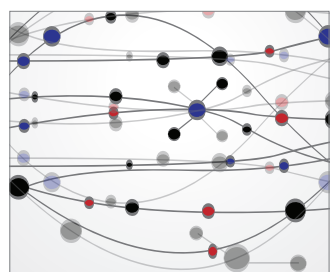

\section{The Scientific} World Journal
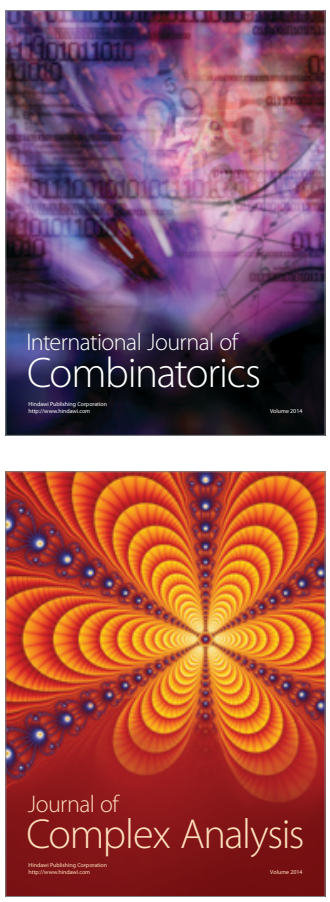

International Journal of

Mathematics and

Mathematical

Sciences
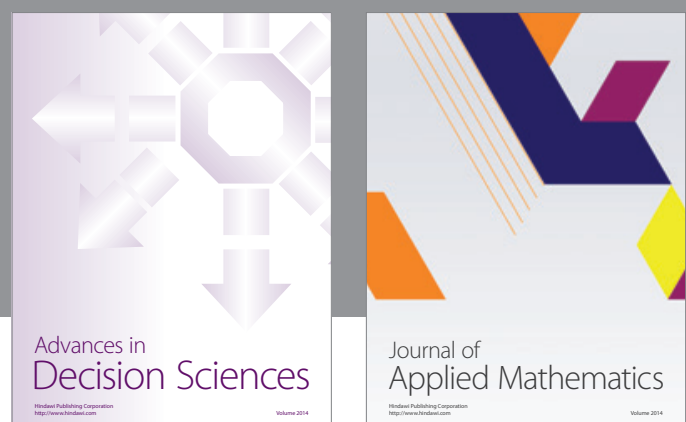

Journal of

Applied Mathematics
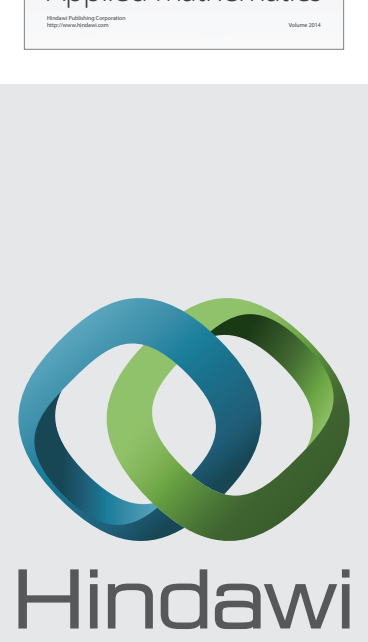

Submit your manuscripts at http://www.hindawi.com
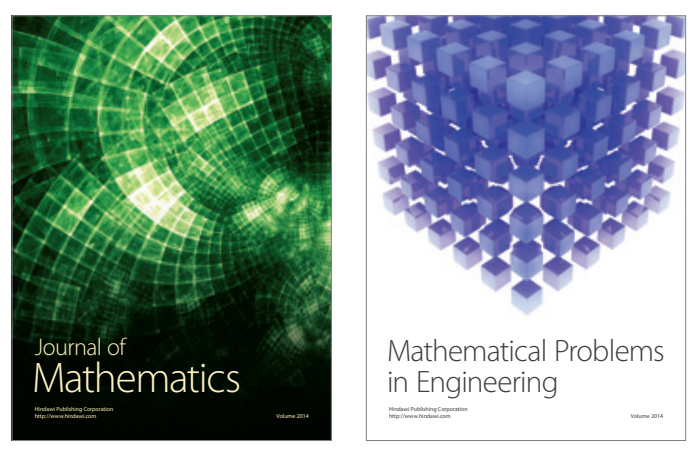

Mathematical Problems in Engineering
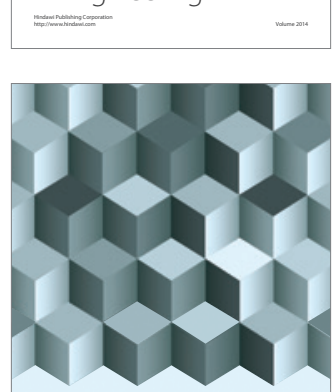

Journal of

Function Spaces
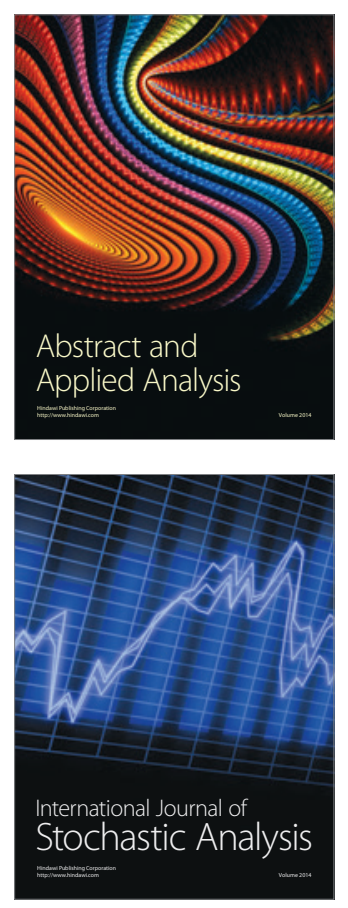

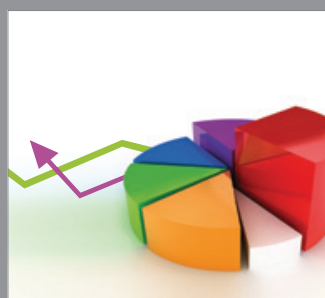

ournal of

Probability and Statistics

Promensencen
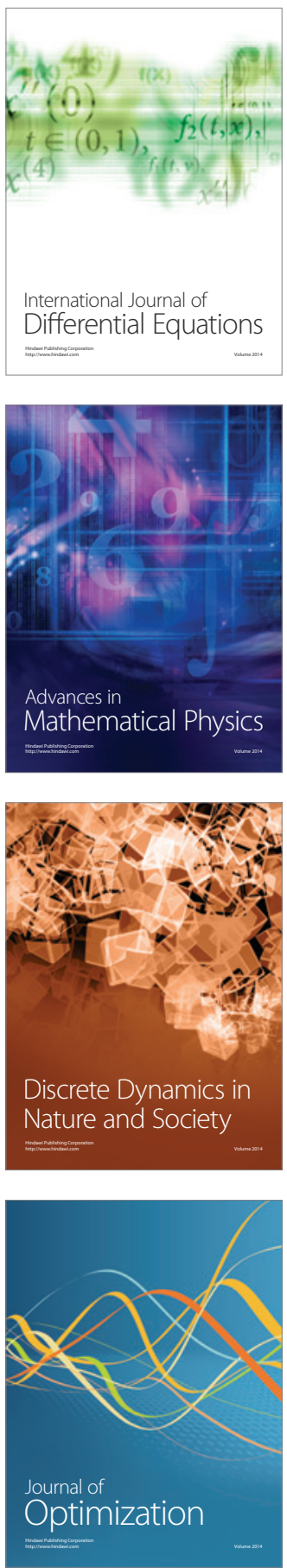